Service social

\title{
Pour des interventions anti-oppressives auprès des jeunes trans : nécessités sociales, évidences scientifiques et recommandations issues de la pratique
}

Denise Medico et Annie Pullen-Sansfaçon

Volume 63, numéro 2, 2017

Genres et sexualités chez les jeunes

URI : https://id.erudit.org/iderudit/1046497ar

DOI : https://doi.org/10.7202/1046497ar

Aller au sommaire du numéro

Éditeur(s)

École de travail social et de criminologie de l’Université Laval

ISSN

1708-1734 (numérique)

Découvrir la revue

Citer cet article

Medico, D. \& Pullen-Sansfaçon, A. (2017). Pour des interventions anti-oppressives auprès des jeunes trans : nécessités sociales, évidences scientifiques et recommandations issues de la pratique. Service social, 63(2), 21-34. https://doi.org/10.7202/1046497ar
Résumé de l'article

Depuis 2011, de plus en plus d'études documentent les besoins et enjeux des jeunes et des enfants qui se sentent différent.e.s dans leur genre. S'il en ressort qu'illes sont encore parmi les jeunes les plus à risque de suicide, de violence par les pairs, d'échec scolaire, de dépression, d'anxiété et de troubles alimentaires, un milieu de vie qui les accepte et les soutient semble le facteur le plus déterminant pour leur santé tant psychique que relationnelle et somatique et leur insertion sociale et professionnelle. Pour favoriser un développement harmonieux pour les jeunes trans et en questionnement, une perspective anti-oppressive est aujourd'hui nécessaire. 


\title{
Pour des interventions anti-oppressives auprès des jeunes trans: nécessités sociales, évidences scientifiques et recommandations issues de la pratique
}

\author{
MEDICO, Denise, PhD en psychologie, professeure en sexologie
}

PULLEN SANSFAÇON, Annie, T.S, PhD, professeure en travail social

\section{RÉSUMÉ}

Depuis 2011, de plus en plus d'études documentent les besoins et enjeux des jeunes et des enfants qui se sentent différent.e.s dans leur genre. S'il en ressort qu'illes sont encore parmi les jeunes les plus à risque de suicide, de violence par les pairs, d'échec scolaire, de dépression, d'anxiété et de troubles alimentaires, un milieu de vie qui les accepte et les soutient semble le facteur le plus déterminant pour leur santé tant psychique que relationnelle et somatique et leur insertion sociale et professionnelle. Pour favoriser un développement harmonieux pour les jeunes trans et en questionnement, une perspective anti-oppressive est aujourd'hui nécessaire.

Mots-clés : transgenre, non binaire, enfants, adolescent, transaffirmatif, anti-oppressif

\section{SUMMARY}

Since 2011, an upsurge in evidence has been documenting the needs and issues of young people and children who feel different in their gender. If it turns out that they are still among the young people most at risk of suicide, peer violence, school failure, depression, anxiety and eating disorders, the living environment (accepting and supporting) seems the most determining factor for their psychic, relational and somatic health and their social and professional integration. To promote harmonious development for trans and questioning young people, an anti-oppressive perspective is needed today.

Keywords: transgender, non binary, children, adolescent, transaffirmative, anti-oppressive 


\section{INTRODUCTION}

De plus en plus d'études documentent les besoins et enjeux des jeunes et enfants qui se sentent différent.e.s dans leur genre (Owen-Smith et al., 2018 ; White Hughto, Reisner et Pachankis, 2015). II en ressort qu'illes ${ }^{1}$ sont encore parmi les jeunes les plus à risque de suicide, de violence par les pairs, d'échec scolaire, de dépression, d'anxiété et de troubles alimentaires et que des interventions et mesures tant sociales que de santé sont nécessaires pour favoriser leur insertion dans la société. Les données récentes et les nouvelles perspectives en intervention promeuvent une vision non pathologisante, pragmatique et affirmative. Ces perspectives, que nous qualifierons d'anti-oppressives, sont construites sur la démonstration grandissante qu'un milieu de vie qui les accepte et les soutient semble le facteur le plus déterminant pour la santé tant psychique que relationnelle et somatique de ces jeunes et leur insertion sociale et professionnelle (Olson et al., 2016 ; Pullen Sansfaçon et al., sous presse).

La publication de lignes directrices pour l'inclusion des jeunes trans en milieu scolaire (Table nationale de lutte contre l'homophobie et la transphobie, 2017) et les changements de la Charte québécoise des droits et libertés de la personne, ont certainement contribué à une prise de conscience de l'existence et des besoins des jeunes trans. La loi 103, Loi visant à renforcer la lutte contre la transphobie et à améliorer notamment la situation des mineurs transgenres, a ainsi permis une protection accrue des identités et expressions de genre au Québec (par l'ajout de la notion d'identité de genre et d'expression de genre comme des dimensions explicitement protégées par la Charte), mais également une reconnaissance civile de l'existence même des enfants trans au sein de la société québécoise. Ainsi, depuis 2016, la reconnaissance du droit d'obtenir un changement de nom et de mention de sexe sur l'acte de naissance et, sur cette base, sur l'ensemble des pièces d'identité, implique le respect de l'identité de genre réelle des personnes trans. Bien qu'imparfaitement ${ }^{2}$, cela constitue tout de même une avancée majeure en matière de reconnaissance des enfants et des jeunes trans.

Mais qui sont ces jeunes et ces enfants ? Identifier combien de jeunes et d'enfants sont concerné.e.s soulève une autre question : celle de savoir ce que c'est que d'être une personne trans et donc de la façon dont on définit, nomme et qualifie cette expérience tant individuelle que sociale. Des choix non seulement sémantiques mais aussi politiques, théoriques et idéologique influencent la manière de penser la question et donc de la mesurer et d'en évaluer les enjeux (Collin et al., 2016). Le constat que posent les chercheur.e.s est que nous manquons d'outils validés et simples pour évaluer qui sont les personnes trans et non binaires et que leur nombre a été sous-estimé (Reisner et al., 2015). Il semble que les jeunes soient plus nombreuxes à s'identifier comme trans ou non binaires (Herman et al., 2017). Une récente méta-analyse qui s'est penchée sur plusieurs enquêtes estime la population trans adulte à $0,39 \%$ de la population générale, dont plus de $50 \%$ sont de jeunes adultes (Meervijk et Sevelius, 2017). Les données statistiques proviennent pour la plupart de travaux de santé publique qui ont choisi de définir la question trans sur la base de l'auto-identification. Ils regroupent sous l'appellation

\footnotetext{
${ }^{1}$ Nous utiliserons le néologisme « ille » dans une optique de langage inclusif et pour mettre en évidence la complexité des genres en dehors de la binarité.

2 Bien que les changements législatifs récents permettent aux adultes et aux enfants de présenter des demandes de changement de sexe à l'État civil, certains groupes sont toujours laissés pour compte, soit les personnes sans citoyenneté canadienne. En effet, le Code civil continue d'exiger la citoyenneté canadienne pour le changement de mention de sexe. De plus, la question se complique pour les personnes trans non binaires qui se voient contraintes à choisir entre une mention de sexe féminine ou masculine.
} 
«variant dans le genre» plusieurs catégories telles que «transgenre », «queer » et «en questionnement sur le genre ». Les études de prévalence les plus importantes vont toutes dans le même sens depuis 2014, ce qui nous fait dire qu'un changement social se dessine. Ainsi, dans une étude sur un échantillon représentatif aléatoire de 8166 jeunes du secondaire en Nouvelle-Zélande, $1,2 \%$ ont dit être « transgenres » et $2,5 \%$ ont dit être « en questionnement » sur le genre (Clark et al., 2014). Une nouvelle enquête américaine, pour sa part, avance le chiffre de 2,7\% pour le nombre de jeunes s'identifiant comme trans et fluides dans le genre (trans and gender non-conforming) (Rider et al., 2018). Face à ces changements sociaux importants, il nous semble nécessaire de faire le point sur les connaissances actuelles, tant de terrain (empirique) que de recherche, et de proposer sur cette base des recommandations pour les travailleurs sociaux.

\section{MÉTHODOLOGIE}

Ce texte propose un dialogue entre professions et expériences, entre le travail social et la sexologie, entre deux professionnelles impliquées dans l'intervention de terrain, la recherche communautaire et le développement de ressources et de politiques adaptées aux besoins des personnes vulnérables. Les auteures défendent une perspective située et anti-oppressive, non pas de personnes trans ou non binaires, mais d'alliées, de personnes engagées auprès des communautés depuis plusieurs années. Nous avons d'une part croisé nos expériences du terrain dans le développement de ressources associatives et par les pairs, l'animation de groupes de soutien et l'accompagnement clinique. Nous avons ensuite mis cela en perspective avec l'avancée des connaissances sur les enfants et jeunes trans et non binaires issues des travaux de recherche et des publications francophones et anglophones. Nous avons donc mené une revue de littérature sur ce qui a été publié dans les champs de la santé publique, du travail social, de la psychologie, de la sociologie en anglais et en français avec les mots clés «transgender », « youth », « children », «transgenre», «jeunes», «enfants». «trans*». Cela nous a permis d'identifier un changement majeur de paradigme de pensée que nous situons autour de 2011 et qui se traduit par l'avènement de postures cliniques, de recherche, conceptuelles de type affirmatif et non plus coercitif. C'est un changement qui a déjà été relevé par Pyne (2014) et qui plaide pour une perspective anti-oppressive dans l'amélioration des conditions de vie des jeunes et enfants trans.

\section{NÉCESSITÉS, DONNÉES PROBANTES, RECOMMANDATIONS}

Une perspective anti-oppressive, c'est-à-dire qui vise à contester et à changer les structures d'oppression et de domination dans une visée de justice sociale (Pullen Sansfaçon, 2013), pour être efficace doit s'ancrer dans une compréhension des différents niveaux impliqués et agir à chacun d'eux. Ce qui signifie que nous devons : 1. comprendre l'expérience de vie des enfants et des jeunes dans leur développement et leur diversité ; 2. ensuite comprendre leurs besoins spécifiques pour développer des ressources adaptées ; 3. prendre en compte l'aspect familial et social, soit le contexte de vie des enfants et des jeunes ; 4. connaître et appliquer les standards de soin ; 5 . agir au niveau plus social et politique pour développer une société plus inclusive.

Nous avons pris le parti de diviser notre propos à partir de ces différents niveaux et pour chacun de relever nos expériences de terrain et ce que la littérature nous indique, de résumer les données actuelles et de proposer, le cas échéant, des recommandations. 


\section{Comprendre les jeunes d'un point de vue développemental et dans leur diversité}

Le vécu des jeunes trans n'est pas monolithique et leurs trajectoires, leurs identités et la manière dont illes manifestent extérieurement et vivent intérieurement leur différence de genre sont très individuelles (Pullen Sansfaçon, 2015). Pour comprendre ce que vit un.e enfant ou un.e jeune qui est confronté.e à des questionnements sur son genre, qui sent que celui qui lui a été assigné à la naissance ne lui convient pas, il faut minimalement penser en fonction de son développement affectif, cognitif et sexuel et en fonction des particularités de son milieu de vie.

Dans les travaux quantitatifs qui ne ciblent pas spécifiquement des personnes trans, environ la moitié des jeunes qui se disent trans rapportent s'être senti.e.s différent.e.s dans leur genre bien avant l'adolescence (Clark et al., 2014). Dans cette étude, qui nous semble particulièrement pertinente, 27,3\% ont ressenti cette différence avant l'âge de 8 ans, 17,9\% entre 8 et 11 ans et 54,8 \% à 12 ans ou plus. Toutefois, lorsque les échantillonnages portent directement sur les personnes trans, ce qui semble se dégager est que le développement d'un sentiment d'identité de genre différent apparaît très tôt, parfois dès 3-4 ans (Ehrensaft, 2011), mais généralement entre 5 et 8 ans (Olson, Key et Eaton, 2015).

Si la compréhension intérieure (celle que l'enfant a pour soi et de soi) est précoce chez la plupart, le moment de la révélation à l'entourage est généralement plus tardif et certains travaux placent même à la fin de l'adolescence, vers 17 ans, l'âge moyen de la révélation à l'entourage (Olson et al., 2015). II est donc primordial de différencier compréhension et expression du sentiment de genre. Si la plupart des jeunes savent qu'illes sont différent.e.s dans leur genre, illes ne l'expriment pas forcément et le gardent même souvent secret. En effet, dès l'âge préscolaire les enfants comprennent les stéréotypes de genre et la pression à s'y conformer (Carver, Yunger et Perry, 2003). Beaucoup, surtout lorsque ce sentiment devient clair entre 6 et 12 ans, lorsque la socialisation hors de la famille est plus importante, auront peur d'être rejeté.e.s ou menacé.e.s s'illes en parlent ou le montrent (Riley et al., 2013). Ainsi, $65,2 \%$ des jeunes trans ou en questionnement ne l'avaient dit à personne avant de répondre à l'étude de Clark et al. (2014).

Si la révélation sociale risque d'entraîner rejet et violence par les pairs ou le milieu de vie, le problème de l'invisibilité et du silence est celui du secret. Vivre dans le secret a des conséquences psychologiques et relationnelles en termes d'isolement, de solitude, de manque de ressources et d'estime de soi (Guédeney, 2011) qui apparaissent dans les suivis que nous faisons dans les cliniques. Nous pouvons constater dans notre expérience de terrain que certains jeunes qui n'expriment pas à leur entourage leur malaise identitaire développent des comportements à risque, de la dépression, des comportements autodestructeurs et de l'agressivité. La situation s'améliore lorsque ces jeunes établissent un lien de confiance avec un adulte et expriment leurs questionnements sur le genre et l'ampleur de la nonadéquation du genre qui leur a été assigné.

La question de la révélation de soi et de la confiance envers des adultes de référence s'esquisse comme fondamentale dans la pratique clinique et l'intervention en travail social. Différents facteurs peuvent expliquer pourquoi certain.e.s expriment leur genre ou se confient à leur entourage. À des fins de simplification, nous pouvons en identifier trois types. II y a d'abord des facteurs contextuelssystémiques, soit le contexte familial et social, son ouverture réelle et celle perçue par l'enfant, ensuite des facteurs individuels, soit la personnalité de l'enfant plus ou moins introvertie et affirmée, et un troisième facteur est le type de rapport au genre et à la sexualité de l'enfant. L'âge est une donnée 
cruciale car il a été démontré depuis longtemps par la psychologie du développement qu'un.e enfant de 4 ans et un.e adolescent.e de 14 n'ont ni la même manière de se penser et d'agir, ni la même compréhension du genre et de la sexualité. Il est peu probable qu'un.e enfant de 4 ans s'identifie comme non binaire, alors que cela est de plus en plus fréquent chez les adolescent.e.s (Steensma et al., 2013). Ainsi, les connaissances actuelles portent majoritairement sur les enfants dont l'expression de genre est de type binaire, c'est-à-dire qui s'identifient assez clairement à un genre masculin ou féminin (Connoly et al., 2016). Ces enfants expriment en général très tôt, parfois dès la petite enfance, être de l'autre genre, vouloir " changer de sexe " ou leur refus des attributs esthétiques et comportementaux du genre qui leur a été assigné. Illes se montrent, dans le vocabulaire utilisé par les professionnel.le.s de la santé mentale, souvent insistant.e.s, persistant.e.s et consistant.e. $s^{3}$ dans leur affirmation de leur appartenance à l'autre genre (Ehrensaft, 2011). Ce sont surtout ces enfants et leurs familles qui vont consulter tôt, soit avant la puberté, et qui bénéficieront d'un accompagnement médical au moment de la puberté.

D'autres enfants et adolescent.e.s se sentent différent.e.s dans leur genre mais d'une autre manière. Par exemple, dans la recherche de Pullen Sansfaçon et ses collègues (sous presse) auprès de jeunes trans, les mots utilisés par celleux-ci pour définir comment illes s'identifient étaient presque aussi nombreux que les participant.e.s elleux-mêmes. Le concept de genre ne leur correspond pas, voire ne fait pas sens. Ainsi certain.e.s peuvent se sentir neutres dans leur genre et ne se sentir correspondre ni à la féminité ni à la masculinité. Illes pourront s'identifier plus tard comme agenrés ou « neutres dans le genre ». Pendant l'enfance illes passeront souvent inaperçu.e.s, resteront invisibles aux yeux de leur famille et de leur entourage (Schneider, 2013), surtout s'illes sont assignés au féminin (considéré.e.s appartenir au genre féminin).

Une autre manière de vivre le genre qui commence à être de plus en plus visible est la non-binarité de genre. Le sentiment de genre ne correspond ni au féminin ni au masculin, mais à une forme d'entredeux, d'alternance ou aux deux à la fois (Richards et al., 2016). Cette expérience du genre se manifeste généralement plus tardivement, soit à l'adolescence (Steensma et al., 2013). Dans les cohortes de jeunes LGBT et queers, plus de $20 \%$ des jeunes ne se définissent pas dans une configuration binaire (auto-identification) et les personnes à qui le genre féminin a été assigné seraient d'ailleurs plus nombreuses (Bosse et Chiodo, 2016 ; Richards et al., 2016).

\section{Comprendre les besoins spécifiques pour développer des ressources adaptées}

Il a été établi de manière consensuelle que les difficultés affectives, la dépression, l'anxiété, les symptômes post-traumatiques, les troubles alimentaires et les idéations suicidaires sont beaucoup plus fréquents chez les jeunes trans (Clark et al., 2014 ; Connolly et al., 2016 ; Grossman, Park et Russell, 2016). En contexte scolaire, le harcèlement, les insultes et la violence sont fréquents pour tous les jeunes LGBT, mais il semble que plus un.e jeune est non conforme au genre qui lui a été assigné, plus ille est susceptible de subir de la violence et des abus ainsi que de la cyberintimidation (Clark et al., 2014 ; Johnson, Singh et Gonzalez, 2014). Ces expériences négatives auraient des répercussions à

\footnotetext{
${ }^{3}$ Ce qui signifie globalement qu'illes ne changent pas d'avis durant leur enfance et insistent sur le fait que le genre qui leur a été assigné n'est pas le bon. Cela a été un des critères majeurs dans les procédures diagnostiques menant aux traitement médical endocronologique par retards de puberté.
} 
l'âge adulte, tant sur la santé mentale que sur l'avenir professionnel et économique (Lenning et Buist, 2012).

Les études les plus récentes semblent indiquer que ces difficultés ne sont pas intrinsèques à une identité trans ni ne joueraient un rôle causal contribuant à l'adoption d'un genre trans, mais sont bien une conséquence directe et indirecte des discriminations, des violences, des mauvaises relations avec les pairs, de la non-reconnaissance de l'identité trans et des pressions à cacher son sentiment de soi (Connoly et al., 2016 ; Raymond et al., 2015; White Hughto et al., 2015). En faveur de cette compréhension systémique, les travaux psychologiques qui ont étudié les fonctions cognitives et l'adaptation affective des enfants trans vivant ouvertement leur genre désiré avec des parents qui les soutiennent montrent qu'illes ont les mêmes aptitudes que les enfants qui ne sont pas transgenres (dits cisgenres). Ainsi Olson et ses collègues $(2016$; 2015) mènent en ce moment des travaux aux ÉtatsUnis sur les enfants trans de 5 à 12 ans qui vivent dans des familles qui leur permettent de vivre dans le genre désiré et qui les soutiennent dans l'espace social. Ces études montrent que les enfants ne sont pas confus.e par rapport à leur genre, ne se différencient pas des enfants cisgenres et ont une santé mentale pratiquement identique. Leur niveau d'anxiété n'est que marginalement plus élevé. Ce genre de recherche nous indique que lorsque les milieux de vie soutiennent les jeunes trans dans leur identité réelle, leur épanouissement, leur développement et leur santé sont meilleurs. On constate que le taux de suicide, qui est un aspect crucial, diminue de $93 \%$ chez les jeunes qui bénéficient d'un soutien fort de la part de leurs parents (Travers et al., 2012). Si encore peu d'études ont clairement démontré des liens causaux entre les environnements oppressifs et les difficultés vécues, le modèle du stress minoritaire, pour sa part, met en avant que les environnements hostiles nuisent à la santé des personnes LGBT (Meyer, 2003). Ce qui suggèrerait que les expériences de victimisation, d'oppression et de discrimination vécues par les jeunes trans nuisent à leur développement optimal. Les pistes d'intervention doivent donc non seulement soutenir les jeunes, mais également leur famille et leur entourage. Elles doivent s'inscrire dans une vision plus structurelle et politique en facilitant les changements nécessaires pour rendre la société plus inclusive.

\section{Prendre en compte le contexte de vie : familles et modèles positifs}

Pour se défendre et contrecarrer la victimisation, les jeunes ont besoin de modèles positifs, de relations harmonieuses avec leurs pairs et de familles « soutenantes » et soutenues (Pullen Sansfaçon et al., sous presse). Les personnes trans plus âgées qui n'ont pas eu la possibilité de mettre des mots sur leur réalité, de comprendre ce qu'elles vivaient et d'avoir des modèles positifs rapportent avoir vécu de la détresse jusqu'au moment où la révélation à soi a pu se faire. Elles disent combien le manque de modèles positifs et de confiance dans leur entourage a été nuisible (Medico, 2016 ; Morgan et Stevens, 2012). À l'inverse, les groupes de pairs sont reconnus comme des facteurs de résilience pour les jeunes trans et en questionnement et leurs familles (Menvielle et Rodnan, 2011).

Le soutien familial est donc un des aspects les plus fondamentaux pour le bien-être des jeunes trans (McConnell, Birkett et Mustanski, 2016), mais il doit lui aussi être soutenu et accompagné. Le cheminement de tous les membres de la famille vers l'acceptation peut être difficile, prendre du temps, commencer par un sentiment de chocs et entraîner des conflits entre les parents comme au sein de la sphère familiale ; il implique souvent un processus d'adaptation (Pullen Sansfaçon et al., 2015) ou de deuil (Wahlig, 2015). Toutefois, le sentiment dominant des parents d'enfants trans semble être le désir de protéger leur enfant contre les violences et les discriminations sociales (Pullen Sansfaçon, 
Robichaud et Dumais-Michaud, 2015 ). Ainsi, décider d'autoriser son enfant à exprimer son identité de genre choisie à l'extérieur de la sphère familiale implique souvent une anxiété et un profond questionnement sur ce qui constitue la «bonne décision » (Pullen Sansfaçon et al., 2015). Lorsqu'on travaille directement auprès des parents et des enfants concernés, il ressort qu'au Québec l'accès aux ressources reste un enjeu majeur et que des différences importantes selon les régions, urbaines ou rurales, ainsi qu'en fonction des ressources financières des parents sont à déplorer sur les terrain et au quotidien.

\section{4. État actuel des pratiques en santé et données probantes sur les meilleures pratiques}

Les approches d'accompagnement dans une optique transaffirmative, c'est-à-dire qui soutiennent les jeunes et les enfants dans leur genre désiré, et proposées à temps, soit avant la puberté, se sont révélées apporter des améliorations importantes dans la qualité de vie et le fonctionnement psychosocial des jeunes trans (Olson et al., 2015, 2016 ; Tishelman et al., 2015). Un changement de paradigme est en ce moment reconnu et encouragé par les associations professionnelles telles que l'APA et l'Association canadienne des travailleurs sociaux (Pullen Sansfaçon, 2015), qui se positionnent clairement dans cette optique transaffirmative. Étant donné l'affluence des demandes, le développement de centres de soins est réclamé par la plupart des spécialistes (Wiepjes et al., 2018). Même le DSM, en adoptant une nouvelle définition, a mis l'accent sur la souffrance associée à la réalité des personnes trans et non plus sur la non-conformité aux normes de genre. De même les approches cliniques recommandées ont abandonné la tentative de "guérison » et de reconversion, pour favoriser un accompagnement et un encadrement visant à faire diminuer la discrimination, à renforcer les liens sociaux et à développer l'affirmation de soi (APA, 2015). Les thérapies qui tentaient de dissuader les jeunes d'exprimer leur genre se sont montrées inutiles, maltraitantes, iatrogènes et non éthiques (APA, 2015). Les jeunes ayant accès à des services professionnels qui les soutiennent dans leur identité de genre désirée présenteraient moins de problèmes de comportement que les jeunes ayant suivi des thérapies de conversion (Torres et al., 2015). Mais l'accès aux services affirmatifs, même généralistes, reste problématique (Galantino et al., 2017 ; Medico et Volkmar, 2016).

Certains jeunes ont des besoins spécifiques et auront besoin d'un accompagnement médicalisé. Ainsi, une partie des enfants trans souhaitera prendre définitivement un genre social différent et faire une transition hormonale pour que leur corps puisse être en adéquation avec leur genre, avec ou sans chirurgie de confirmation de genre par la suite. Pour ces jeunes, le moment de la puberté est alors particulièrement crucial puisqu'il est un point de non-retour somatique qui impliquera par la suite de lourdes interventions médicales pour en corriger les effets (Olson, 2016 ; Vance, Ehrensaft et Rosenthal, 2014).

Les traitements hormonaux de blocage de puberté ( $\mathrm{GnRH}$ analogues) sont pratiqués depuis plus de vingt ans et ont démontré leur efficacité sur le plan psychologique et social et leur faisabilité sur le plan somatique (Cohen-Kettenis et Klink, 2015). Ces traitements sont recommandés dans les normes de soins des sociétés scientifiques internationales comme la World Professional Association for Transgender Health (WPATH, 2012), l'American Academy of Pediatrics (2017) et l'American Society of Endocrinology (2017) pour les enfants et les jeunes qui veulent vivre dans l'autre genre de manière cohérente et sans signe extérieur qui pourrait les stigmatiser. Ce traitement permet de suspendre pour quelques mois ou quelques années leur développement pubertaire afin de leur permettre d'atteindre un âge suffisant et la maturité psychique nécessaire pour prendre une décision éclairée sur leur genre 
définitif. Ce traitement est prescrit aux jeunes à partir du moment où illes ont atteint un stade de Tanner 2, soit au début de la puberté et en fonction de la maturité de l'enfant (Cohen-Kettenis et Klink, 2015). Des thérapies dites croisées sont également disponibles et souhaitables ; il s'agit alors de déclencher la puberté dans le genre désiré en favorisant ainsi un développement harmonieux, au même âge que les autres, et une parfaite invisibilité sociale (lorsqu'elle est désirée). Des services novateurs intégrant différents champs professionnels et travaillant dans des perspectives transaffirmatives se développent depuis plusieurs années. Mais le problème reste l'accès aux services : trop rares, trop distants, trop chers pour certaines familles (Pullen Sansfaçon et col 2015), car ils sont situés uniquement dans certains grands centres urbains et qu'en dehors des traitements prodigués par les médecins et psychiatres participant au régime public, qui ont par ailleurs de longues listes d'attente, les services en psychothérapie sont privés et donc payants. C'est pourquoi une action sur le plan politique et social est nécessaire, dans une perspective anti-oppressive qui ne situe pas les problèmes dans l'individu mais dans son rapport aux systèmes.

\section{Agir au niveau social et politique pour instaurer une société équitable}

Au-delà des interventions individualisées, l'amélioration des conditions de vie des jeunes trans ne peut donc se faire sans passer par le développement de politiques et de lois qui favorisent tant leur inclusion que leur protection. Les problèmes qu'illes vivent sont causés par l'absence généralisée d'acceptation au sein de la société (Ghosh, 2012) qui souvent comprend leur vécu comme un caprice, une situation marginale ou un problème de santé mentale. Pourtant, plusieurs s'entendent pour dire que la pathologisation de l'identité de genre chez les enfants est un phénomène culturel, puisqu'on ne remarque que très peu de souffrance chez les populations qui affirment le genre de l'enfant ou le laissent explorer librement son identité de genre (Suess Schwend et al., 2018). Ainsi, une première étape vers une société plus inclusive à l'égard des personnes trans doit passer par un accroissement de la conscience sociale et la dépathologisation de leur existence (Winter et al., 2016). Selon nous, cela n'est réellement possible qu'à travers la création de lois et de politiques sociales permettant explicitement l'expression de toutes les identités de genre.

Par exemple, la société étant largement construite autour du modèle binaire, les jeunes sont socialisés dans un contexte qui leur donne peu de choix. Ainsi les jeunes trans non binaires doivent continuer de s'identifier aux deux seuls marqueurs de sexe autorisés actuellement. Pour favoriser une reconnaissance de toustes, peu importe le genre, de type binaire ou non, il serait bon de retirer la mention du sexe sur les papiers d'identité, par exemple. Car dans les faits une identité civile non congruente avec l'expression visible du genre est un obstacle majeur à l'intégration pour les personnes trans.

La création d'un espace de vie respectueux de tous passe aussi par le développement d'initiatives concrètes servant à garantir les droits des jeunes trans. Si l'identité de genre et l'expression de genre sont maintenant protégées au Québec par la Charte des droits et libertés de la personne, la pratique peut être différente. Dans les écoles, les jeunes trans se voient encore dénier l'accès aux toilettes de leur choix ou imposer une mention de sexe qui ne leur correspond pas. Les personnes trans migrantes ne peuvent toujours pas se prévaloir des mêmes droits que les personnes ayant la citoyenneté. Sachant que les jeunes trans vivent de la détresse psychologique et une faible estime personnelle, il importe d'assurer que les droits et libertés soient garantis systématiquement dans l'ensemble des espaces qui les accueillent et qu'illes n'aient pas à porter constamment la charge de les faire respecter. 
Des lignes directrices, un message clair et des pistes d'intervention pour protéger les droits et libertés de tous sont nécessaires. Par exemple, l'adoption des lignes directrices Mesures d'ouverture et de soutien envers les jeunes trans et les jeunes non binaires (Table nationale de lutte contre l'homophobie et la transphobie, 2017) pour l'inclusion des jeunes trans dans les écoles contribue certainement à assurer que leurs droits soient respectés et que les services soient offerts dans tous les milieux, ruraux ou urbains. Cela dit, afin de favoriser l'inclusion et la protection complète des droits des jeunes trans dans la société civile, il importe que les initiatives découlent du gouvernement et que les balises soient à portée nationale. Par exemple, des lignes directrices ministérielles pour le milieu de l'éducation, tant primaire qu'universitaire, permettraient d'éviter que les directions d'établissements puissent décider de ce qui est acceptable ou non, et diminueraient le fardeau pour les jeunes trans de devoir défendre leurs droits auprès de leur établissement scolaire. Le nouveau Plan de lutte contre l'homophobie et la transphobie 2016-2022 (Gouvernement du Québec, 2017) semble aller dans ce sens sur certains aspects, cependant, il n'est pas suffisamment clair sur la manière dont il répond à d'autres enjeux touchant aux questions de citoyenneté, ainsi que sur le problème des pièces d'identité, qui actuellement ne peuvent pas être changées au Québec.

\section{Conclusion}

II est de plus en plus clair, dans la littérature scientifique comme sur le terrain, que c'est le fait de se sentir trans et/ou non binaire et d'être identifié.e ainsi, de ne pas s'accepter, de ne pas être accepté.e et de subir de la violence qui vulnérabilise les enfants et les jeunes trans. Cela induit des conséquences néfastes et à long terme sur la santé mentale et physique et sur les conditions de vie en général. De plus, les jeunes trans semblent bien plus nombreuxes que les anciennes données l'indiquaient. L'état actuel de la recherche nous indique assez clairement les interventions à favoriser pour offrir aux enfants et jeunes trans un avenir meilleur que celui qui a été largement dépeint dans les dernières décennies. Cela implique la lutte contre la discrimination et le harcèlement en milieu scolaire pour tous les jeunes différents dans leur genre et en questionnement, le soutien aux ressources communautaires, psychologiques et médicales transaffirmatives ; et pour les enfants et jeunes qui ont besoin d'une prise en charge plus médicale au moment de la puberté, un accès à des ressources qui doivent travailler en fonction des dernières normes de soins établies par la World Professional Association for Transgender Health (WPATH, 2012) et dans une optique transaffirmative.

Illes ont besoin d'un environnement favorable, aidant et sécurisant pour grandir. II est donc fondamental de développer des ressources et des politiques pour les protéger dans leurs milieux de vie. Les ressources doivent être conscientes de l'importance du contexte et des effets systémiques et doivent aussi avoir une vision fluide du genre. Elles doivent aussi prendre en compte la diversité des expériences et l'intersectionnalité, soit le croisement de plusieurs facteurs de vulnérabilité comme par exemple le niveau socioéconomique ou le fait d'être racisé.e. Dans ce contexte, les travailleurs sociaux, les éducateurs, les enseignants et le système scolaire ont un rôle crucial à jouer, en prenant en compte ces divers facteurs et en adaptant leurs pratiques. Tout cela nous ramène à la nécessité de changements sociaux et politiques. Parmi les jeunes trans, un certain nombre vont également avoir besoin de prises en charge spécialisées, voire de traitements hormonaux, à la puberté. Devant les résultats très encourageants des normes de soins actuels, des projets de recherche sont en cours au Canada, aux États-Unis et en Europe afin d'étudier les effets de l'accompagnement par des cliniques aux pratiques transaffirmatives et dans des milieux de vie qui les acceptent et les appuient. Les premiers 
résultats semblent indiquer que ces enfants ne se différencient plus des autres enfants, qu'illes vont bien, sont heureuxes et envisagent l'avenir avec plus de confiance.

MEDICO, Denise, Ph. D. en psychologie, professeure en sexologie Université du Québec à Montréal

PULLEN SANSFAÇON, Annie, T.S, PhD, professeure en travail social Université de Montréal

\section{RÉFÉRENCES}

American Academy of Psychiatry (AAP) (2017). Statement on protecting transgender youth. [https://www.aap.org/en-us/about-the-aap/aap-press-room/pages/AAP-Statement-on-ProtectingTransgender-Youth.aspx]

American Psychological Association (APA) (2015). Guideline for psychological practice with transgender and gender non conforming people. [http://www.apa.org/practice/guidelines/transgender.pdf]

Bosse, J. D., et Chiodo, L. (2016). It is complicated: Gender and sexual orientation identity in LGBTQ youth. Journal of Clinical Nursing, vol. 25, nos 23-24, p. 3665-3675.

Carver, P. R., Yunger, J. L., et Perry, D. G. (2003). Gender identity and adjustment in middle childhood. Sex Roles, vol. 56, p. 439-448.

Clark, T. C., Lucassen, M. F. G., Bullen, P., et al. (2014). The health and well-being of transgender high school students: Results from the New Zealand Adolescent Health Survey (Youth'12). Journal of Adolescent Health, vol. 55, n 1, p. 93-99.

Cohen-Kettenis, P. T., et Klink, D. (2015). Adolescents with gender dysphoria. Best Practice \& Research - Clinical Endocrinology \& Metabolism, vol. 29, nº 3, p. 485-495.

Collin, L., Reisner, S. L., Tangpricha, V., et Goodman, M. (2016). Prevalence of transgender depends on the "case" definition: A systematic review. Journal of Sexual Medicine, vol. 13, n 4, p. 613-626.

Connolly, M. D., Zervos, M. J., Barone, C. J., Johnson, C. C., et Joseph, C. L. M. (2016). The mental health of transgender youth: Advances in understanding. Journal of Adolescent Health, vol. 59, $n^{\circ} 5$, p. 489-495.

Ehrensaft, D. (2011). Gender born, gender made: Raising healthy gender-nonconforming children. New York: The Experiment.

Galantino, G., Blais, M., Hébert, M., et Lavoie, F. (2017). Un portrait de l'environnement social et de l'adaptation psychosociale des jeunes québecois.e.s trans identifié.e.s ou en questionnement de 
leur identité de genre. Rapport de recherche du projet Parcours amoureux des jeunes LGBT du Québec. Montréal : Université du Québec à Montréal.

Ghosh, S. (2012). Gender identity. Medscape, 11 juin 2012.

[http://emedicine.medscape.com/article/917990-overview]

Gouvernement du Québec (2017) Plan d'action gouvernementale de lutte contre l'homophobie et la transphobie 2017-2022. En ligne.

[https://www.justice.gouv.qc.ca/fileadmin/user_upload/contenu/documents/Fr_francais_centredoc /publications/ministere/plans-

actions/Plan_action_gouvernemental_lutte_contre_homophobie_transphobie_2017-2022.pdf ] [dernier accès 7 mars 2018]

Grossman, A. H., Park, J. Y., et Russell, S. T. (2016). Transgender youth and suicidal behaviors:

Applying the interpersonal psychological theory of suicide. Journal of Gay \& Lesbian Mental Health, vol. $20, n^{\circ} 4$, p. 329-349.

Guédeney, N. (2011). Les racines de l'estime de soi. Devenir, vol. 23, p. 129-144.

Herman, J., Flores, A. R., Brown, T. N. T., Wilson, B. D. M, et Conron, K. (2017). Age of individuals who identify as transgender in the United States. Los Angeles : The Williams Institute, UCLA School of Law. [https://williamsinstitute.law.ucla.edu/wp-content/uploads/TransAgeReport.pdf].

Johnson, C. W., Singh, A. A., et Gonzalez, M. (2014). "It's complicated": Collective memories of transgender, queer, and questioning youth in high school. Journal of Homosexuality, vol. $61, \mathrm{n}^{\circ} 3$, p. 419-434.

Lenning, E., et Buist, C. L. (2013). Social, psychological and economic challenges faced by transgender individuals and their significant others: Gaining insight through personal narratives. Culture, Health \& Sexuality, vol. 15, n 1, p. 44-57.

McConnell, E. A., Birkett, M., et Mustanski, B. (2016). Families matter: Social support and mental health trajectories among lesbian, gay, bisexual, and transgender youth. Journal of Adolescent Health - Official Publication of the Society for Adolescent Health and Medicine, vol. 59, n 6, p. 674680.

Medico, D. (2016.) Repenser le genre. Une clinique avec les personnes trans*. Genève : Georg éditeur, coll. « Médecine Société (CMS)》.

Medico, D., et Volkmar, E. (2016). La Fondation Agnodice. Pour une société plus juste à l'égard des personnes transgenres. Nouvelles Questions Féministes, vol. 35, n 1, p. 182-186.

Meervijk, E., et Sevelius, J. M. (2017). Transgender population size in the United States: A metaregression of population-based probability samples. American Journal of Public Health, vol. 107, $\mathrm{n}^{\circ} 2$, e1-e8. 
Menvielle, E. J., et Rodnan, L. A. (2011). A therapeutic group for parents of transgender adolescents. Child and Adolescent Psychiatric Clinics of North America, vol. 20, nº 4, p. 733-743.

Meyer, I. H. (2003). Prejudice, social stress, and mental health in lesbian, gay, and bisexual populations: Conceptual issues and research evidence. Psychological Bulletin, vol. 129, n 5 , p. 674-697.

Morgan, S. W., et Stevens, P. E. (2012). Transgender identity development as represented by a group of transgender adults. Issues in Mental Health Nursing, vol. 33, n 5, p. 301-308.

Olson, K. R. (2016). Prepubescent transgender children: What we do and do not know. Journal of the American Academy of Child \& Adolescent Psychiatry, vol. 55, n 3, p. 155-156.

Olson, K. R., Key, A. C., et Eaton, N. R. (2015). Gender cognition in transgender children. Psychological Science, vol. 26, n 4 , p. 467-474.

Olson, K. R., Durwood, L., DeMeules, M., et al. (2016). Mental health of transgender children who are supported in their identities. Pediatrics, vol. 137, p. 1-8.

Owen-Smith, A. A., Gerth, J., Sineath, R. C., et al. (2018). Association between gender confirmation treatments and perceived gender congruence, body image satisfaction and mental health in a cohort of transgender individuals. Journal of Sexual Medicine, 17 février 2018. doi : 10.1016/j.jsxm.2018.01.017

Pullen Sansfaçon, A. (2015). Parentalité et jeunes transgenres : un survol des enjeux vécus et des interventions à privilégier pour le développement de pratiques transaffirmatives. Santé mentale au Québec, vol. 40, n³, p. 93-107.

Pullen Sansfaçon, A. (2013). La pratique anti-oppressive (p. 353-373). Dans H. Dorvil et E. Harper (dir.), Le travail social. Théories, méthodologies et pratiques. Québec : Presses de l'Université du Québec.

Pullen Sansfaçon, A., et Bellot, C. (2017). L'éthique de la reconnaissance comme posture pour travailler avec les jeunes trans. Nouvelles pratiques sociales, vol. $28, n^{\circ} 2$, p. 38-53.

Pullen Sansfaçon, A., Robichaud, M.-J., et Dumais-Michaud, A.-A. (2015). The experience of parents who support their children's gender variance. Journal of LGBT Youth, vol. 12, $\mathrm{n}^{\circ}$ 1, p. 39-63.

Pullen Sansfaçon, A., Ward, D., Dumais-Michaud, A.-A., Robichaud, M.-J, et Clegg, A. (2014). Working with parents of gender variant children: Using social action as an emancipatory research framework. Journal of Progressive Human Services, vol. 25, n 3 , p. 214-229.

Pullen Sansfaçon, A., Hébert, W., Lee, O., Faddoul, M., Tourki, D., et Bellot, C. (sous presse). Digging beneath the surface: Results from stage one of a qualitative analysis of factors influencing the wellbeing of trans youth in Quebec. International Journal of Transgenderism. doi :

$10.1080 / 15532739.2018 .1446066$ 
Pyne, J. (2014). Gender independent kids: A paradigm shift in approaches to gender non-conforming children. Canadian Journal of Human Sexuality, vol. 23, n²1, p. 1-8.

Raymond, G., Blais, M., Bergeron, F.-A., et Hébert, M. (2015). Les expériences de victimisation, la santé mentale et le bien-être de jeunes trans au Québec. Santé mentale au Québec, vol. 40, n 3, p. 77-92.

Reisner, S. L., Vetters, R., Leclerc, M., et al. (2015). Mental health of transgender youth in care at an adolescent urban community health center: A matched retrospective cohort study. Journal of Adolescent Health, vol. 56, n 3, p. 274-279.

Richards, C., Bouman, W. P., Seal, L., Barker, M. J., Nieder, T. O., et T'Sjoen, G. (2016). Non-binary or genderqueer genders. International Review of Psychiatry, vol. 28, n 1, p. 95-102.

Rider, G. N., McMorris, B. J., Gower, A. L., et al. (2018). Health and care utilization of transgender and gender nonconforming youth: A population-based study. Pediatrics, vol. 141, $\mathrm{n}^{\circ} 3$. doi : 10.1542/peds.2017-1683

Riley, E. A., Clemenson, L., Sitharthan, G., et Diamond, M. (2013). Surviving a gender-variant childhood: The views of transgender adults on the needs of gender-variant children and their parents. Journal of Sex and Marital Therapy, vol. 39, n³, p. 241-263.

Schneider, E. (2013). Les droits des enfants intersexes et trans' sont-ils respectés en Europe ? Une perspective. Rapport présenté au Conseil de l'Europe, novembre 2013. [http://itgl.lu/wpcontent/uploads/2015/04/Les-droits-des-enfants-intersexes-et-trans-sont-ils-respect \%C3 \%A9s-enEurope_-Une-perspective.pdf] [accès 27/02 2018]

Steensma, T. D., McGuire, J. K., Kreukels, B. P. C., Beekman, A. J., et Cohen-Kettenis, P. T. (2013). Factors associated with desistence and persistence of childhood gender dysphoria: A quantitative follow-up study. Journal of the American Academy of Child and Adolescent Psychiatry, vol. 52, $n^{\circ} 6$, p. 582-590.

Suess Schwend, A., Winter, S., Chiam, Z., Smiley, A., et Cabral Grinspan, M. (2018). Depathologising gender diversity in childhood in the process of ICD revision and reform. Global Public Health. doi : 10.1080/17441692.2018.1427274

Table nationale de lutte contre l'homophobie et la transphobie dans le réseau de l'éducation (2017) Mesures d'ouverture et de soutien envers les jeunes trans et les jeunes non-binaires. En ligne [http://www.colloquehomophobie.org/wp-content/uploads/2018/01/Brochure-Jeunes-TransWeb.pdf][dernier accès 7 mars 2018]

Tishelman, A. C., Kaufman, R., Edwards-Leeper, L., et al. (2015). Serving transgender youth: Challenges, dilemmas and clinical examples. Professional Psychology - Research and Practice, vol. $46, n^{\circ} 1$, p. 37-45. 
Torres, C. G., Renfrew, M., Kenst, K., et al. (2015). Improving transgender health by building safe clinical environments that promote existing resilience: Results from a qualitative analysis of providers. BMC Pediatrics, vol. 15, n 187. doi : 10.1186/s12887-015-0505-6

Travers, R., Bauer, G., Pyne, J., Bradley, K., pour le Trans PULSE Project (2012). Impacts of strong parental support for trans youth: A report prepared for Children's Aid Society of Toronto and Delisle Youth Services. Toronto : Trans PULSE.

Vance, S. R., Ehrensaft, D., et Rosenthal, M. (2014). Psychological and medical care of gender nonconforming youth. Pediatrics, vol. 134, p. 1184-1192.

Wahlig, J. L. (2015). Losing the child they thought they had: Therapeutic suggestions for an ambiguous loss perspective with parents of a transgender Child. Journal of GLBT Family Studies, vol. $11, n^{\circ} 4$, p. 305-326.

White Hughto, J. M., Reisner, S. L., et Pachankis, J. E. (2015). Transgender stigma and health: A critical review of stigma determinants, mechanisms, and interventions. Social Science \& Medicine, vol. 147, p. 222-231.

Wiepjestal, C. M., et al. (2018). The Amsterdam cohort of gender dysphoria study (1972-2015): Trends in prevalence, treatment, and regrets. The Journal of Sexual Medicine, 17 février. doi : 10.1016/j.jsxm.2018.01.016

Winter, S., Ehrensaft, D., Pickstone-Taylor, S., De Cuypere, G., et Tando, D. (2016). The psychomedical case against a gender incongruence of childhood diagnosis. The Lancet Psychiatry, vol. 3, $\mathrm{n}^{\circ} 5$, p. 404-405.

World Professional Association for Transgender Health (WPATH) (2012). Standards of care (SOC) for the health of transsexual, transgender, and gender nonconforming people - Version 7. International Journal of Transgenderism, vol. 13, n 4, p. 165-232. 